\title{
SMART DISPLAY BOARD WITH CCTV FOR ATTENDANCE
}

Luv Mahalwal ${ }^{1}$, Jhanvi Gulati ${ }^{2}$, Latika Duhan ${ }^{3}$, Antim Dev Mishra ${ }^{4}$

E-Mail Id: ${ }^{1}$ luvmahalwal07@gmail.com, ${ }^{2}$ jhanvigulati60@gmail.com, ${ }^{3}$ latikaduhan@ ansaluniversity.edu.in, 4antimdevmishra@ansaluniversity.edu.in

School of Engineering and Technology, Ansal University, Gurugram, Haryana India

Abstract- Face detection technologies have made many improvements in a short amount of time. Smart Display Board With CCTV For Attendance is a real-world solution which comes with day to day activities of handling student attendance system. A face recognition-based attendance system is a process of recognizing the students face for taking attendance by using face biometrics based on high - definition monitor video and other information technology. In this project, the camera will be able to find and recognize faces fast and precisely in images. The project is made in such a way that the face of the student can be easily recognized for their attendance so that the attendance database can be easily reflected automatically and if the student is not present a message is sent to his/her parents or guardians.

Keywords: Attendance System, Automated Attendance System, Face Detection, Face Recognition Attendance System, Internet of Things (IoT)

\section{INTRODUCTION}

IoT refers to a network of devices that make the network self-configuring.

An IoT ecosystem consists of web-enabled smart devices that use embedded systems, such as processors, sensors, and communication hardware, to collect, send, and act on data they acquire from their environments. IoT devices share the sensor data they collect by connecting to an IoT gateway or other edge device where data is either sent to the cloud to be analyzed or analyzed locally. Sometimes, these devices communicate with other related devices and act on the information they get from one another. The devices do most of the work without human intervention, although people can interact with the devices -- for instance, to set them up, give them instructions or access the data.

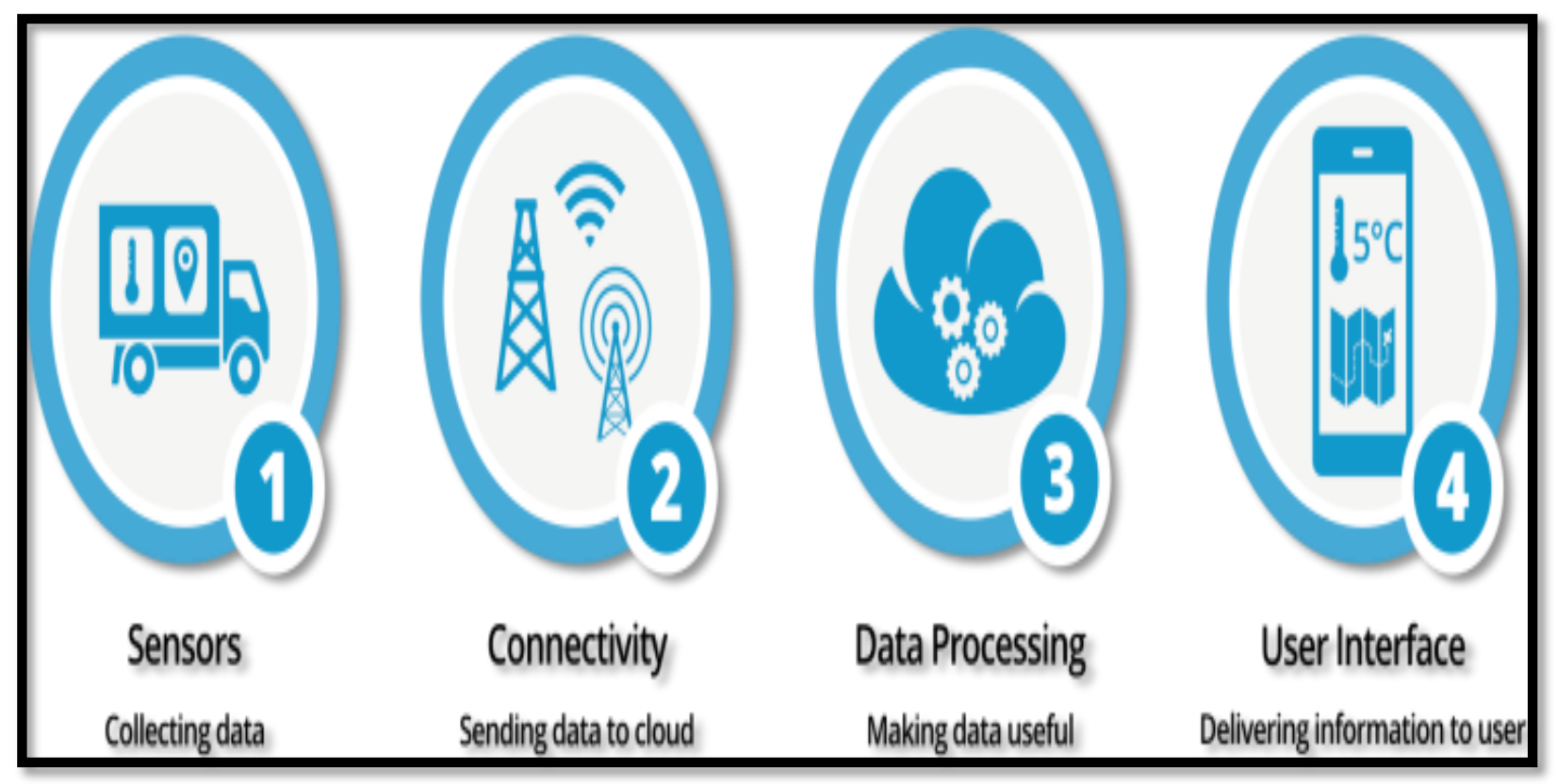

Fig. 1.1 Main Components of An IoT System

\subsection{The 7 Crucial IoT Characteristics}

$>$ Connectivity. This doesn't need too much further explanation. With everything going on in IoT devices and hardware, with sensors and other electronics and connected hardware and control systems there needs to be a connection between various levels.

$>$ Things. Anything that can be tagged or connected as such as it's designed to be connected. From sensors and household appliances to tagged livestock. Devices can contain sensors or sensing materials that can be attached to devices and items.

$>$ Data. Data is the glue of the Internet of Things, the first step towards action and intelligence.

$>$ Communication. Devices get connected so they can communicate data and this data can be analyzed. Communication can occur over short distances or over a long-range to very long range. Examples- Wi- 
$\mathrm{Fi}$, LPWA network technologies such as LoRa or NBIoT.

$>$ Intelligence. The aspect of intelligence as in the sensing capabilities in IoT devices and the intelligence gathered from big data analytics (also artificial intelligence).

$>$ Action. The consequence of intelligence. This can be a manual action, action based upon debates regarding phenomena (for instance in smart factory decisions) and automation, often the most important piece.

$>$ Ecosystem. The place of the Internet of Things from a perspective of other technologies, communities, goals, and the picture in which the Internet of Things fits. The Internet of Everything dimension, the platform dimension, and the need for solid partnerships.

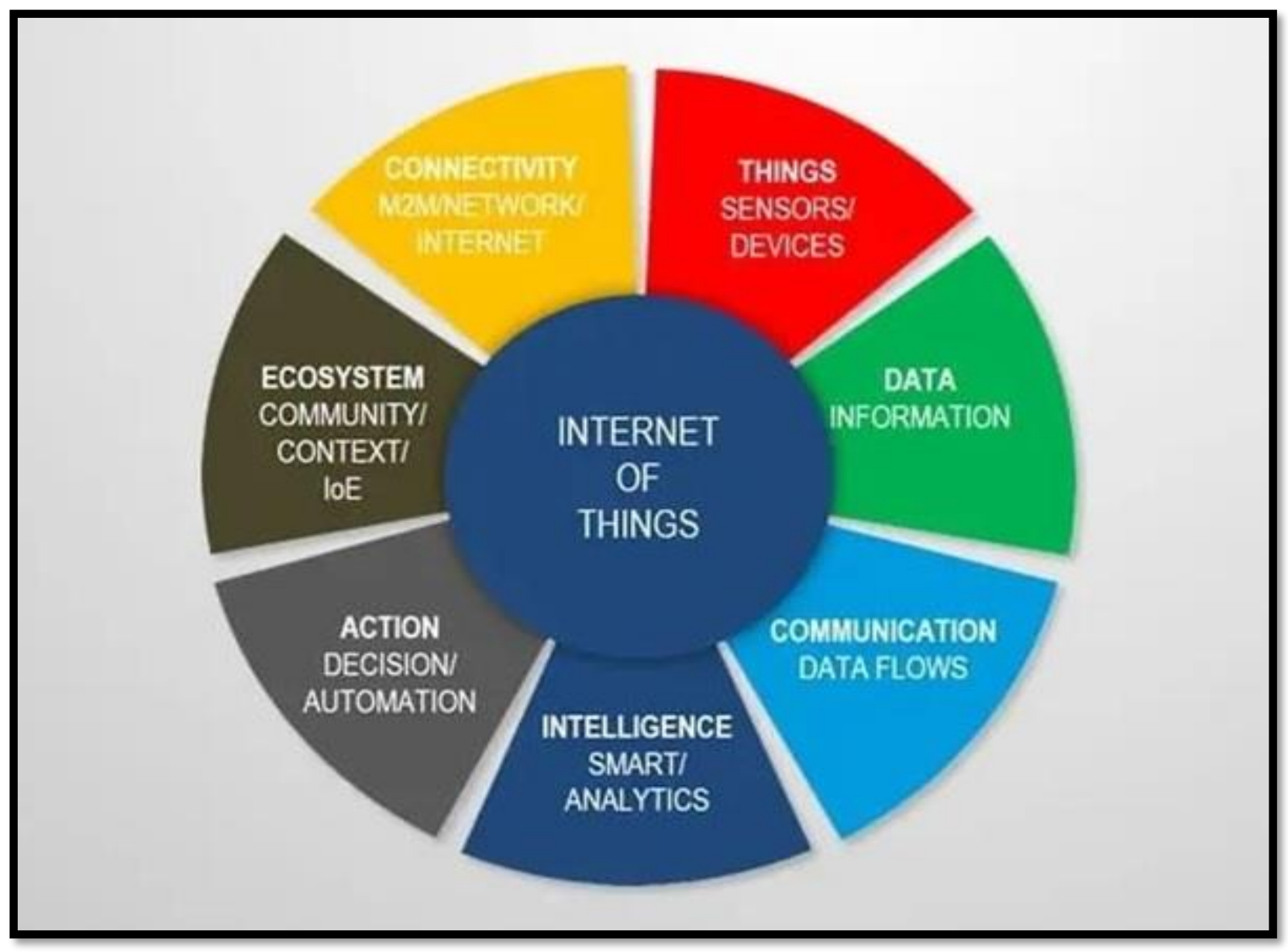

Fig. 1.2 Defining IoT-7 Characteristics

The connectivity, networking, and communication protocols used with these web-enabled devices largely depend on the specific IoT applications deployed. IoT can also make use of artificial intelligence (AI) and machine learning to aid in making data collecting processes easier and more dynamic.

\subsection{IOT Concept And Definition}

The term "Internet of Things" refers to different IoT devices with individual uniqueness and their ability to perform remote sensing, actuation, and live surveillance of certain kinds of data. IoT devices also enable live information sharing with other connected applications and devices, either actively or passively, or data from several other devices to be collected and processed and relayed to different servers. The second-word internet is known as a Global Communication Network that connects the trillions of computers across the globe to allow information sharing. Thus, the IoT can be defined as-

"A dynamic Global Network Infrastructure with self-configuring capabilities based on standard and interoperable communication to a protocol where physical and virtual things have identities, physical attributes, and virtual personalities and use intelligent interfaces and are seamlessly integrated into the information network, often communicate data associated with the user and their environment."

An optimal IoT system consists of multiple interfaces and applications to connect and communicate to other devices/gadgets and can be either wired or wireless. IoT devices can be of many different shapes, such as wearable sensors, smartwatches, IoT smart home monitoring, IoT smart transport systems, IoT smart health devices, etc. 


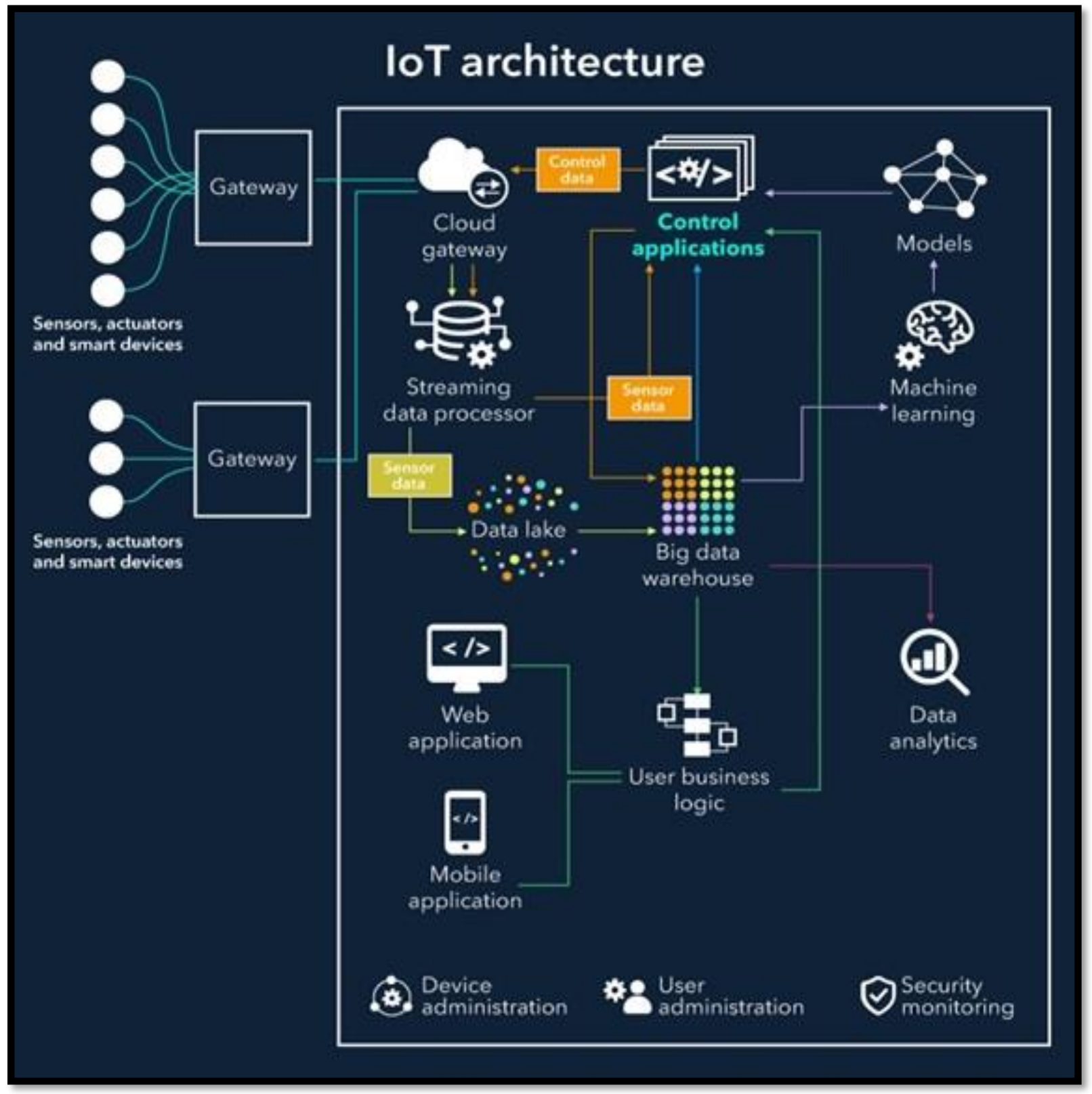

Fig. 1.3 IoT Architecture

IoT enabling technologies - Wireless Sensor Network (WSN)- It consists of different sensors/nodes that are fused or integrated together to track different kinds of data. Cloud Computing-Cloud computing, also known as on-demand computing, is a form of internet-based computing that provides computers and other devices with shared processing resources and data upon demand. It may be in various shapes, such as IaaS, PaaS, SaaS, DaaS, etc.

Since the word "cloud" is often used as an Internet metaphor, "cloud computing" refers to being able to obtain computational power via the world wide web instead of traditional systems where computing equipment is physically present at the user's premises and any software applications are installed on such local hardware. Cloud computing is defined as-

"A model for enabling ubiquitous, convenient, on-demand network access to a shared pool of configurable computing resources (e.g., networks, servers, storage, applications, and services) that can be rapidly provisioned and released with minimal management e ort or service provider interaction."

Cloud computing and its 3 distinct service models-Software as a Service (SaaS), Platform as a Service (PaaS) and Infrastructure as a Service (IaaS) -are relevant to IoT because they enable any user with a browser and internet access to turn smart object information into usable knowledge. In other words, cloud computing offers "the virtual utility computing infrastructure that incorporates applications, monitoring devices, storage devices, analytics tools, visualization platforms, and consumer delivery... [to] enable individuals and companies to reach [IoT-enabled] applications on-demand at anytime, anywhere, anywhere." 


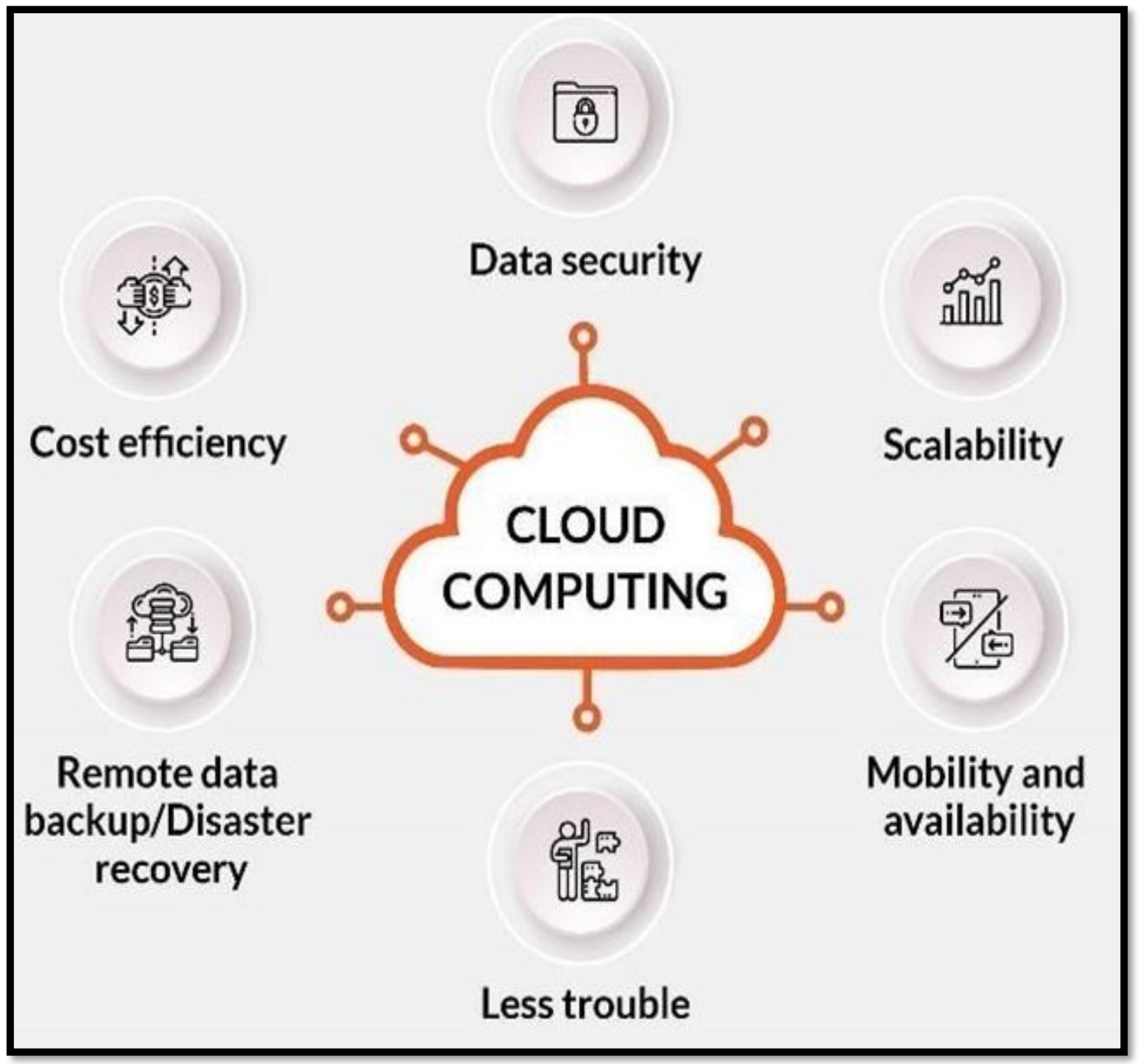

Fig. 1.4 The Advantages of Cloud Computing

\subsubsection{Big Data Analytics}

Big Data-to unmask hidden patterns, unknown correlations, market trends, desires of consumers, and other useful business information. As more intelligent devices (smart objects) are connected to the Internet of Things, more information is gathered from them in order to conduct analytics to identify patterns and affiliations that result in insights.

Thus, the term "big data" refers to certain large sets of data that would need to be processed, preserved, enquired, evaluated, and generally handled in order to deliver on the IoT pledge.

Furthermore, the technological challenges of big data are compounded by the fact that IoT systems must tackle not only the data obtained from intelligent devices but also the spatial information required to conduct such analytics properly (e.g., weather-related public and private data sets, GIS, political, seismic, map, GPS, crime, etc.).

Thus, with more connected devices coming online, IoT operators usually use at least three measures to characterize the big data they handle- quantity, speed, and variety.

\subsubsection{Communication Protocols}

They serve as the foundation of IoT network to allow communication and access to applications, and these protocols promote the sharing of data over the network, since these protocols support information exchange formats, data encoding, and addressing.

\subsubsection{Embedded Systems}

It involves microprocessor/microcontroller, RAM / ROM, networking components, I /O systems, and storage devices. 


\section{BIG DATA \& ANALYTICS}

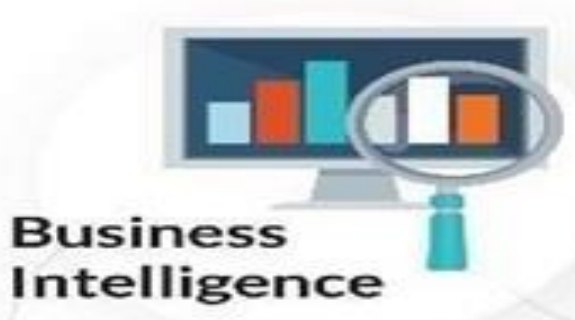

Big Data Analytics services

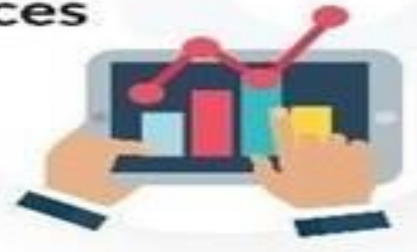

Big data visualization

Fig. 1.5 Big Data and Analytics

\subsection{Deep Learning}

The technology aims in imparting a tremendous knowledge-oriented technical innovation these days. Deep Learning is one of the interesting domains that enables the machine to train itself by providing some datasets as input and provides an appropriate output during testing by applying different learning algorithms. Nowadays Attendance is considered an important factor for both the student as well as the teacher of an educational organization. With the advancement of the deep learning technology the machine automatically detects the attendance performance of the students and maintains a record of those collected data. In general, the attendance system of the student can be maintained in two different forms namely,

> Manual Attendance System (MAS)

$>$ Automated Attendance System (AAS)

Manual Student Attendance Management system is a process where a teacher concerned with the particular subject need to call the students name and mark the attendance manually. Manual attendance may be considered as a timeconsuming process or sometimes it happens for the teacher to miss someone or students may answer multiple times on the absence of their friends. So, the problem arises when we think about the traditional process of taking attendance in the classroom. To solve all these issues we go with Automatic.

Automated Attendance System (AAS) is a process to automatically estimate the presence or the absence of the student in the classroom by using face recognition technology. It is also possible to recognize whether the student is sleeping or awake during the lecture and it can also be implemented in the exam sessions to ensure the presence of the student. The presence of the students can be determined by capturing their faces on a high-definition monitor video streaming service, so it becomes highly reliable for the machine to understand the presence of all the students in the classroom. The two common Human Face Recognition techniques are-

$>$ Feature-based approach

$>$ Brightness-based approach

The Feature-based approach also known as local face recognition system, used in pointing the key features of the face like eyes, ears, nose, mouth, edges, etc., whereas the brightness-based approach also termed as the global face recognition system, used in recognizing all the parts of the image.

\section{LITERATURE SURVEY}

Automated Attendance System using Face Recognition proposes that the system is based on face detection and 
ICACCG2020 30-31 July, 2020, Ansal University, Gurgaon, India

International Journal of Technical Research \& Science (Special Issue)

ISSN No.:2454-2024 (online) recognition algorithms, which is used to automatically detects the student face when he/she enters the class and the system is capable to marks the attendance by recognizing him. Viola-Jones Algorithm has been used for face detection which detects human face using cascade classifier and PCA algorithm for feature selection and SVM for classification. When it is compared to traditional attendance marking this system saves time and also helps to monitor the students. The purpose of developing a Smart Display board with CCTV for attendance is to computerized the traditional way of taking attendance. Another purpose for developing this software is to generate the report automatically at the end of the session or in the between of the session.

The purpose of developing a Smart Display board with CCTV for attendance is to computerize the traditional way of taking attendance. Another purpose for developing this software is to generate the report automatically at the end of the session or in the between of the session.

In this paper basic problem of student attendance management is defined which is traditionally taken manually by faculty. One alternative to make student attendance system automatic is provided by Computer Vision. In this paper, we review the various computerized system which is being developed by using different techniques. Based on this review a new approach for student attendance recording and management is proposed to be used for various colleges or academic institutes.

\section{Do you like the currently attendance system?}

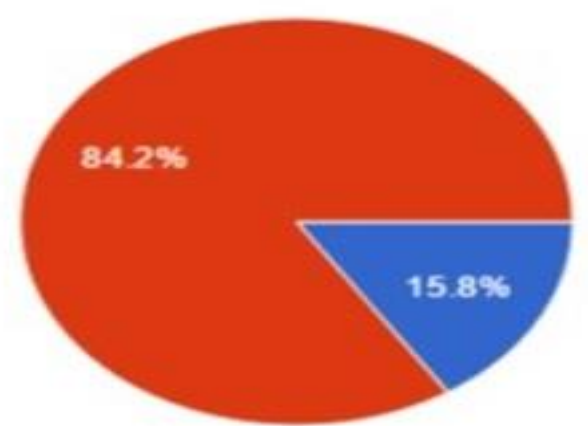

Fig. 2.1 Poll for Current Attendance System

A school, college, or university can save up to thousands of rupees monthly by automating student attendance. If a teacher has 7 lectures a day and requires 10 minutes to mark attendance for each lecture then nearly 70 minutes are consumed in marking attendance. If there are 50 teachers in the institution then each day 58 hours are required for the mundane task of marking attendance. The 58 hours can easily be reduced to 1 hour, and the considerable amount of teachers' time saved can be utilized towards improving student outcomes.

\section{EXISTING RECOGNITION SYSTEMS}

\subsection{Fingerprint Based recognition system-}

In the Fingerprint-based existing attendance system, a portable fingerprint device needs to be configured with the student's fingerprint earlier. Later either during the lecture hours or before, the student needs to record the fingerprint on the configured device to ensure their attendance for the day. The problem with this approach is that during the lecture time it may distract the attention of the students.

\subsection{RFID (Radio Frequency Identification) Based recognition system-}

In the RFID based existing system, the student needs to carry a Radio Frequency Identity Card with them and place the ID on the card reader to record their presence for the day. The system is capable of connecting to RS232 and record the attendance to the saved database. There are possibilities for fraudulent access may occur. Some are students may make use of other student's IDs to ensure their presence when the particular student is absent or they even try to misuse it sometimes.

\subsection{IRIS Based Recognition System}

In the Iris based student attendance system, the student needs to stand in front of a camera, so that the camera will scan the Iris of the student. The scanned iris is matched with data of students stored in the database and the attendance on their presence needs to be updated. This reduces the paper and pen workload of the faculty member of the institute. This also reduces the chances of proxies in the class and helps in maintaining the student records safe. It is a wireless 
ICACCG2020 30-31 July, 2020, Ansal University, Gurgaon, India

International Journal of Technical Research \& Science (Special Issue)

ISSN No.:2454-2024 (online) biometric technique that solves the problem of spurious attendance and the trouble of laying the corresponding network.

\subsection{Face Based Recognition System}

The facial recognition technology can be used in recording the attendance through a high-resolution digital camera that detects and recognizes the faces of the students and the machine compares the recognized face with students' face images stored in the database. Once the face of the student is matched with the stored image, then the attendance is marked in attendance database for further calculation. If the captured image doesn't match with the students' face present in the database then this image is stored as a new image onto the database. In this system, there are possibilities for the camera not to capture the image properly or it may miss some of the students from capturing.

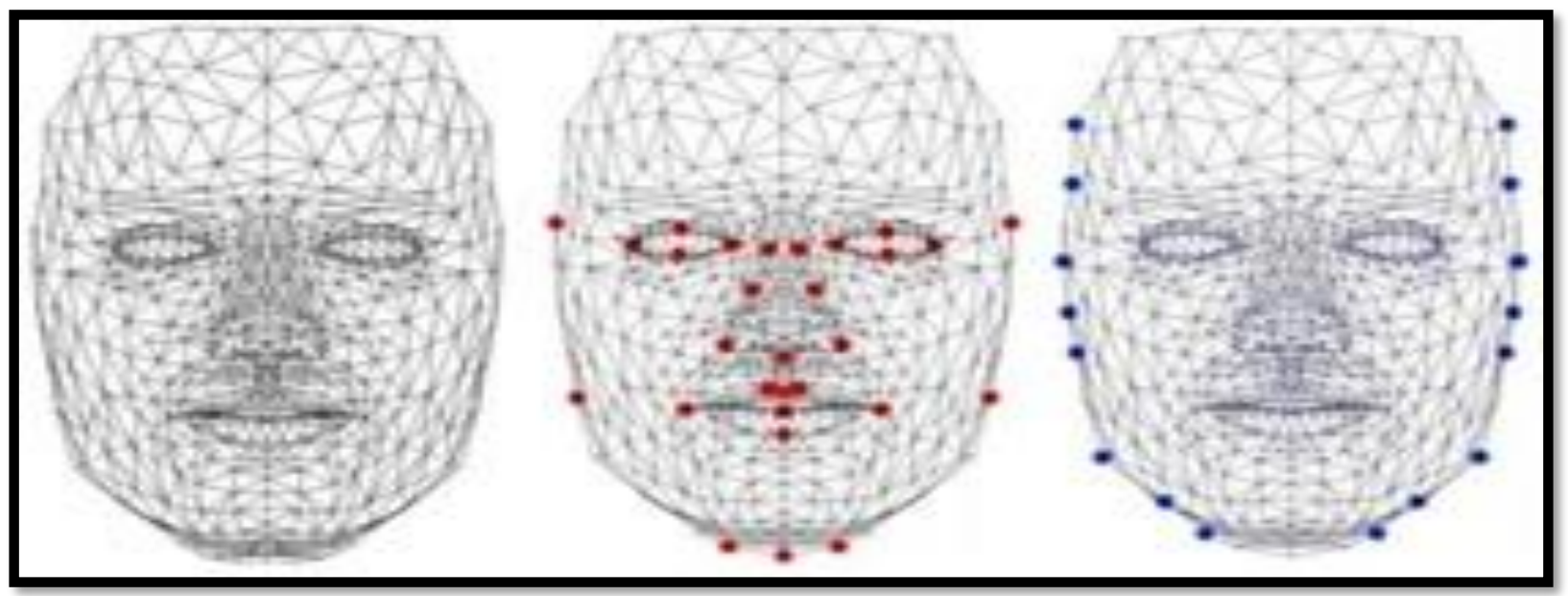

Fig.3.1 Face Alignment Model - Face Image Modeling and Representation

Face recognition may be considered the most secure type of authentication. Because we have an alternative for all the other methods for fake attendance.

\section{PROJECT DESCRIPTION}

\subsection{Definition- IoT based Smart Display board with CCTV for Attendance}

IoT based Smart Display board with CCTV for Attendance is an automated smart attendance system. The raspberry pie is connected to the camera with the help of breadboard and jumping wires. The raspberry pie gets power through the power cable and it is connected with the internet over wifi. The student has to stand in the front of the camera and wait until the camera scans face and compare with faces stored in databases. If the face matches then the student is marked present and if it doesn't match then the student is marked absent and a message is sent to the parents. After that, an excel sheet is generated and it automatically gets uploaded to the server. And the raspberry pie is also connected to the smart display which shows the total class strength, no. of students present, and absent in the class.

Only the lens part of the camera and smart display is visible otherwise all the parts are hidden in a box to make it look stylish, compact, and smart. This can help the teachers by getting better accuracy and if a student tries to show his/her friend's face in the camera it won't detect. So it will protect against fake attendance.

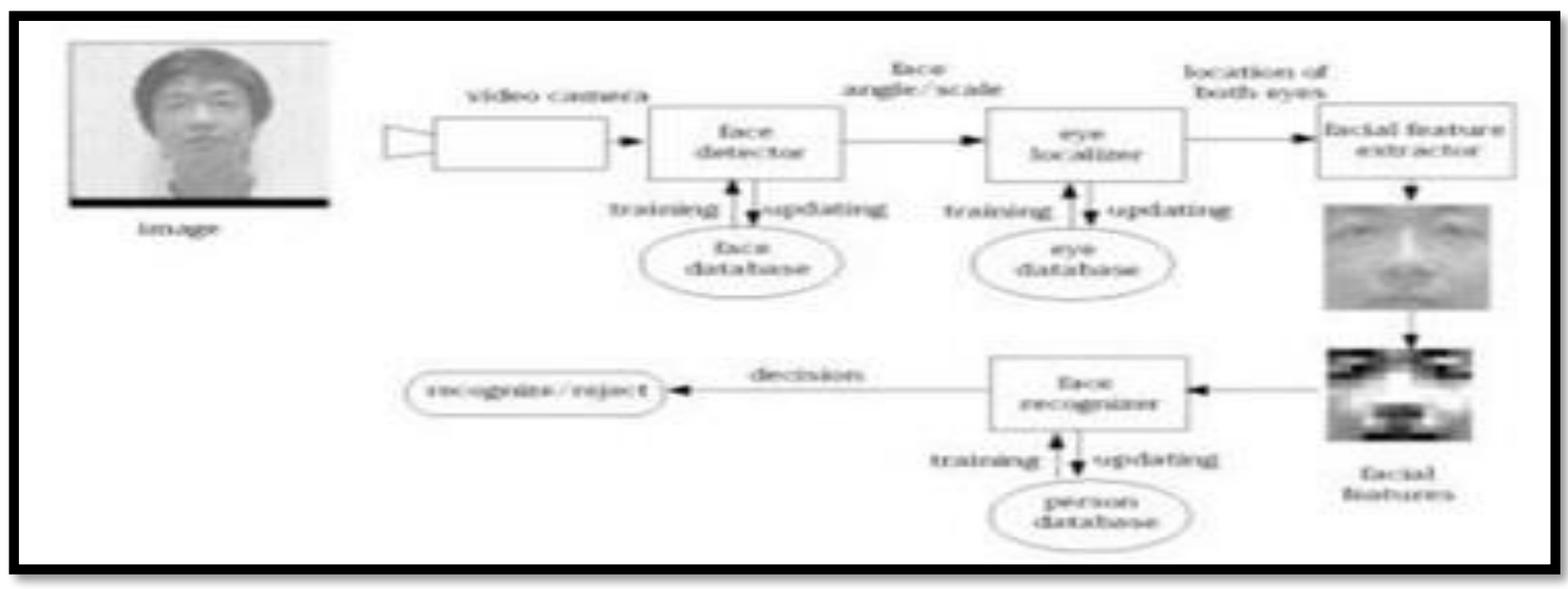

Fig.4.1 Face Recognition System Framework

DOI Number: https://doi.org/10.30780/specialissue-ICACCG2020/0033

Paper Id: IJTRS-ICACCG2020-033

pg. 69 
ICACCG2020 30-31 July, 2020, Ansal University, Gurgaon, India

International Journal of Technical Research \& Science (Special Issue)

\subsection{Components and Modules}

\subsubsection{Raspberry Pi 3}

The Raspberry Pi is a series of small single-board computers developed in the United Kingdom by the Raspberry Pi Foundation

Raspberry Pi 3 Specification-

$>$ Quad Core 1.2GHz Broadcom BCM2837 64bit CPU

$>1 \mathrm{~GB}$ RAM

> BCM43438 wireless LAN and Bluetooth Low Energy (BLE) on board

$>100$ Base Ethernet 40-pin extended GPIO

$>4$ USB 2 ports

$>4$ Pole stereo output and composite video port

$>$ Full-size HDMI

$>$ CSI camera port for connecting a Raspberry Pi camera

$>$ DSI display port for connecting a Raspberry Pi touch screen display

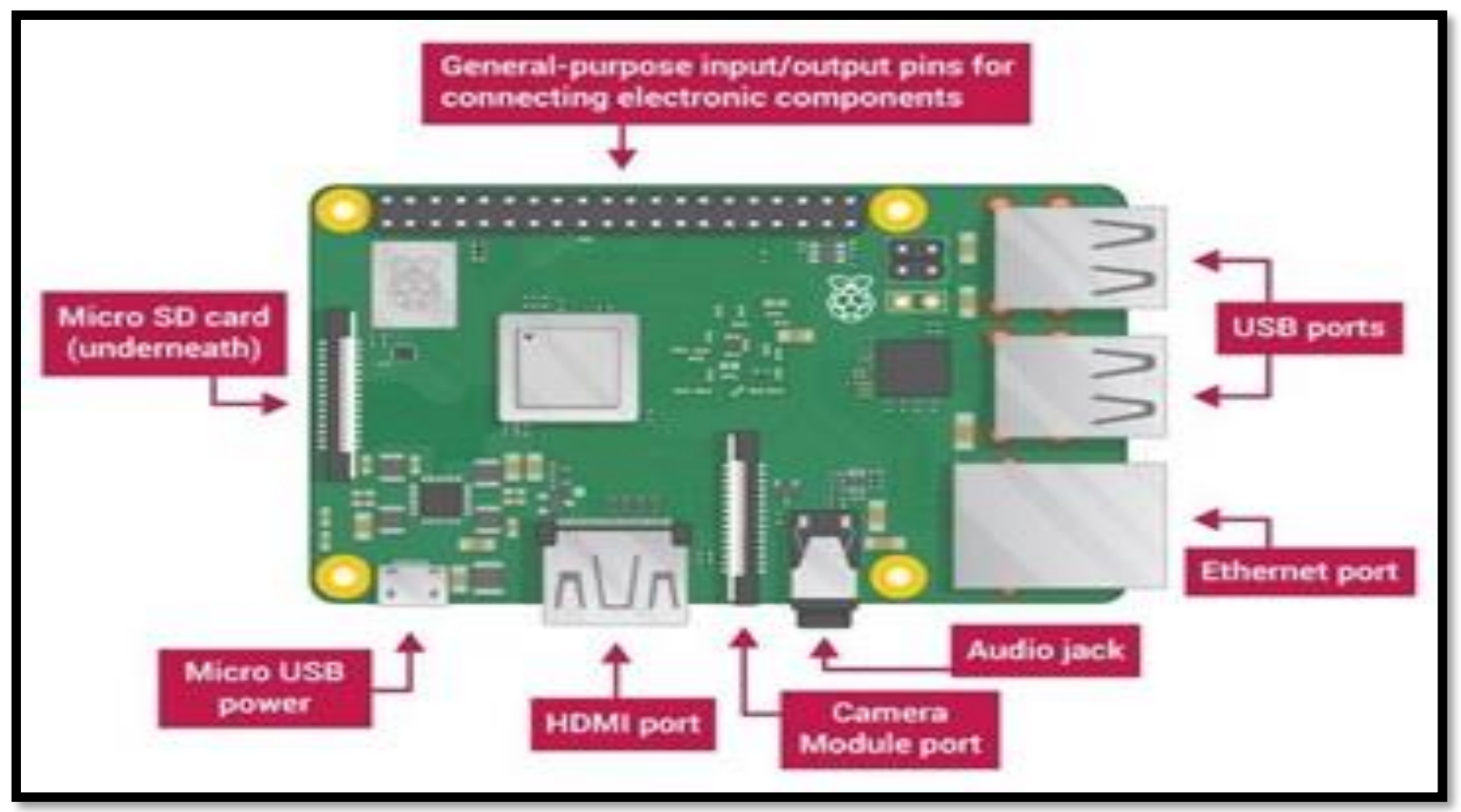

Fig. 4.2 Raspberry Pi 3

\subsubsection{Logitech C930e Webcam}

A webcam is a video camera that feeds or streams an image or video in real-time to or through a computer to a computer network, such as the Internet. Webcams are typically small cameras that sit on a desk, attach to a user's monitor, or are built into the hardware.

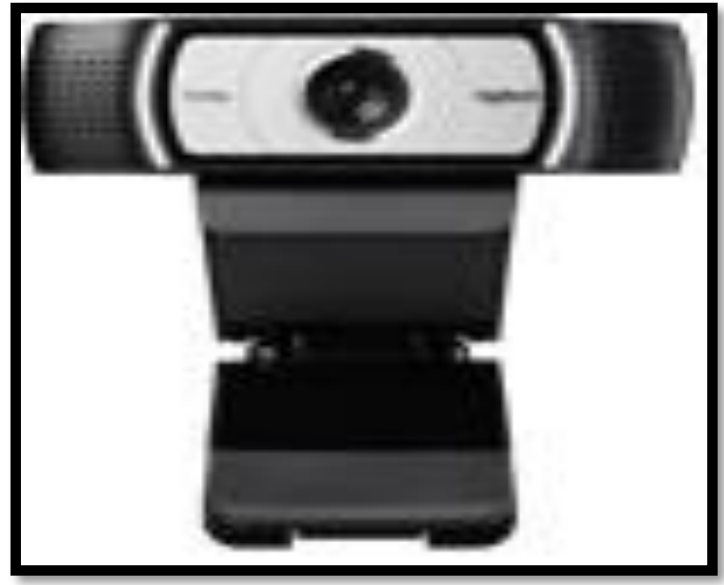

Fig.4.3 Logitech Webcam

DOI Number: https://doi.org/10.30780/specialissue-ICACCG2020/0033 
ICACCG2020 30-31 July, 2020, Ansal University, Gurgaon, India

International Journal of Technical Research \& Science (Special Issue)

ISSN No.:2454-2024 (online)

Web Cam Specifications-

$>$ Full HD 1080p video calling (up to 1920 x 1080 pixels)

$>720$ p HD video calling (up to $1280 \times 720$ pixels) with supported clients

$>$ H.264 video compression

$>90^{\circ}$ field of view

$>$ Zoom to $4 \mathrm{X}$ in $1080 \mathrm{p}$

Night light 2 Technology for clarity in various lighting environments, even low light

\subsubsection{LED Display Board}

A LED display is a flat panel display that uses an array of light-emitting diodes as pixels for a video display. Their brightness allows them to be used outdoors where they are visible in the sun for store signs and billboards.

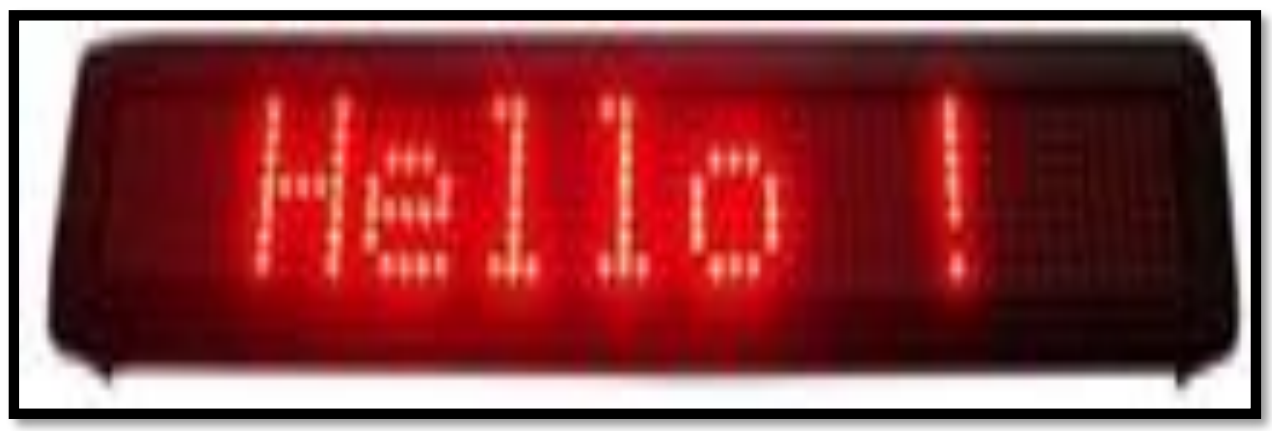

Fig. 4.4 LED Display Board

\subsubsection{Bread Board And Connecting Wires}

A breadboard is utilized to build and test circuits expeditiously afore finalizing any circuit design. The breadboard has many apertures into which route components like ICs and resistors can be connected. The apertures are generally spaced 0.1 " apart to put up standard DIP machinery. Jump wires are generally used to establish connectivity with the breadboard.

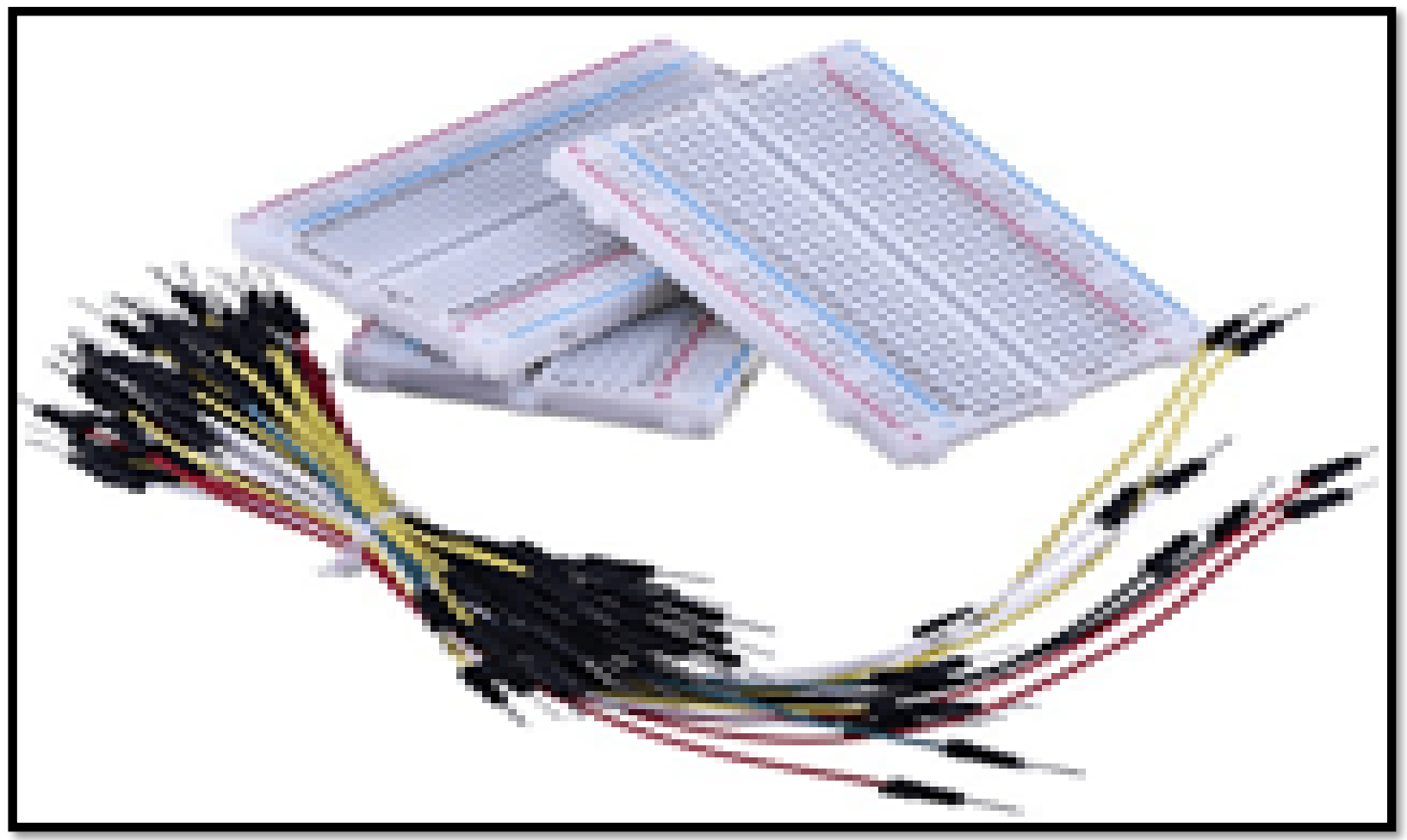

Fig. 4.5 Bread Board and Connecting Wires

\section{PROPOSED WORKING MODEL}

The aim of our dissertation is to provide an attendance system using face detection and recognition on the Raspberry Pi board and send an alert message to the authorized person via mobile device or internet server. This will increase 
ICACCG2020 30-31 July, 2020, Ansal University, Gurgaon, India

International Journal of Technical Research \& Science (Special Issue)

ISSN No.:2454-2024 (online)

the security of our Dissertation. Whatever the system that we have to implement it should work in real-time with a low false detection rate. The block diagram for real-time face detection and recognition system that will contain various hardware and software components. Proposed work diagram of Face Detection and Recognition.

The proposed work follows-

The first part was booting the Raspberry Pi board by installing the Operating system Raspbian OS and installing the essential libraries and packages.

$>$ Interfacing of a camera module with a raspberry pi B3 model. Capture face image using USB Webcam i.e. image acquisition take place.

$>$ Create a database of authorized persons using the face detection process. After creating a database save into folder.

$>$ Capture current face, compare with database image. Using face detection and face recognition process. Algorithms are used for face detection and recognition.

$>$ If any person is not present then the message will be sent to the absent person.

$>$ Excel sheet is generated and uploaded automatically on the server.

$>$ Faculty can access the excel sheet using mobile or pc.

\section{1 Circuit Diagram}

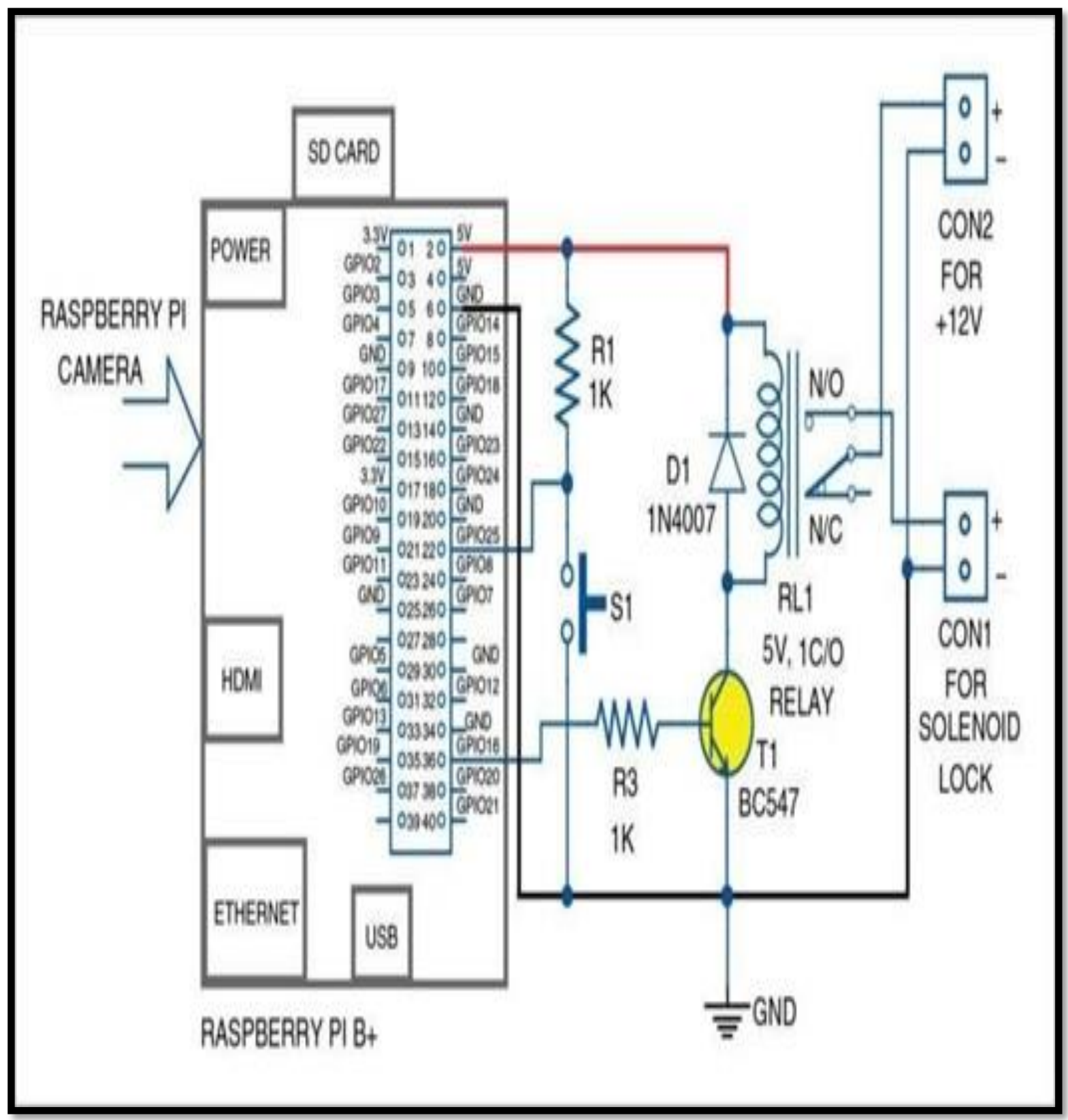

Fig..5.1 Circuit Diagram of Proposed Working Model

DOI Number: https://doi.org/10.30780/specialissue-ICACCG2020/0033 
ICACCG2020 30-31 July, 2020, Ansal University, Gurgaon, India

International Journal of Technical Research \& Science (Special Issue)

5.2 Flowchart

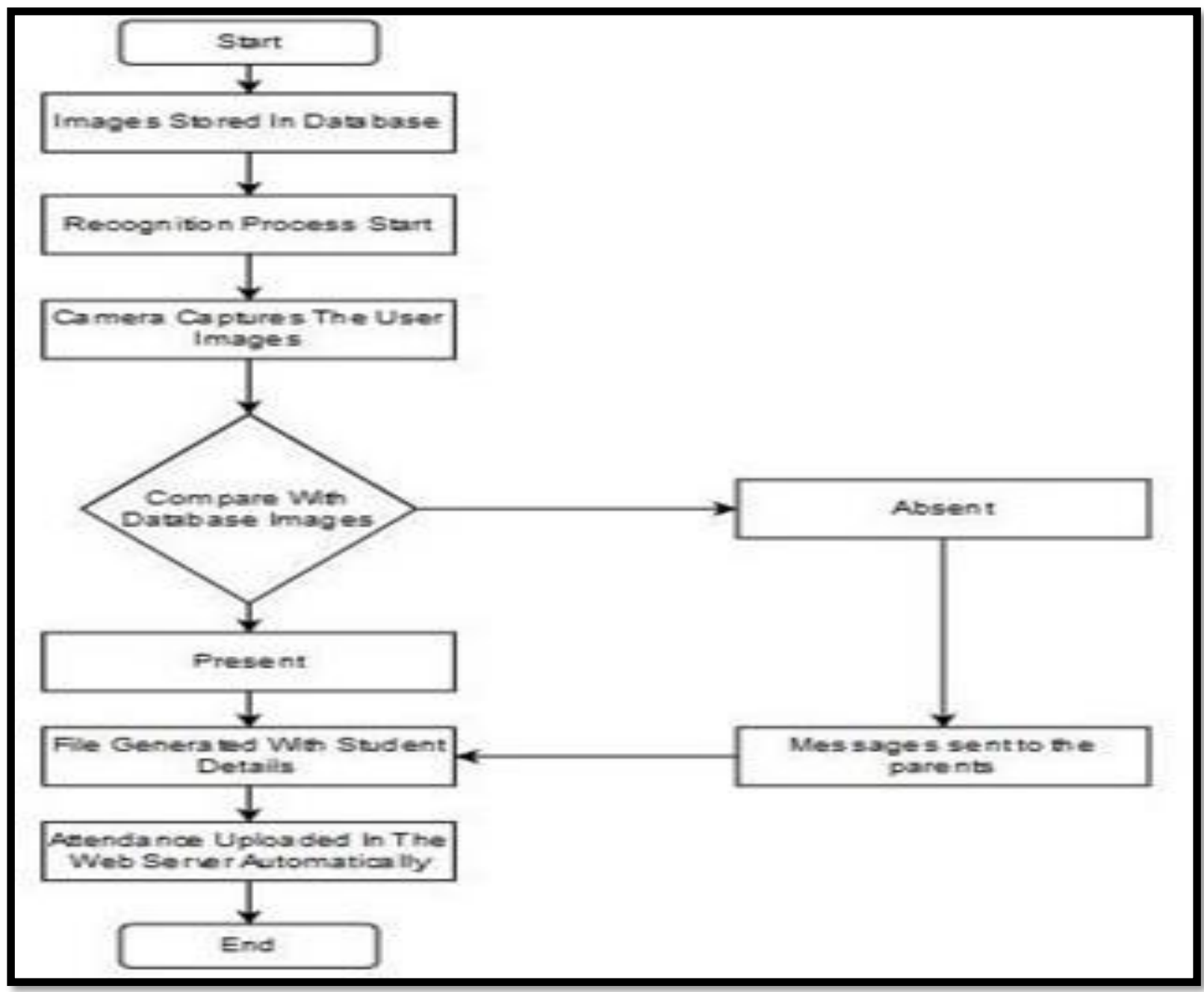

Fig.5.2 Flowchart Of Proposed Working Model

5.3 Face Detection and Recognition Flow Diagram

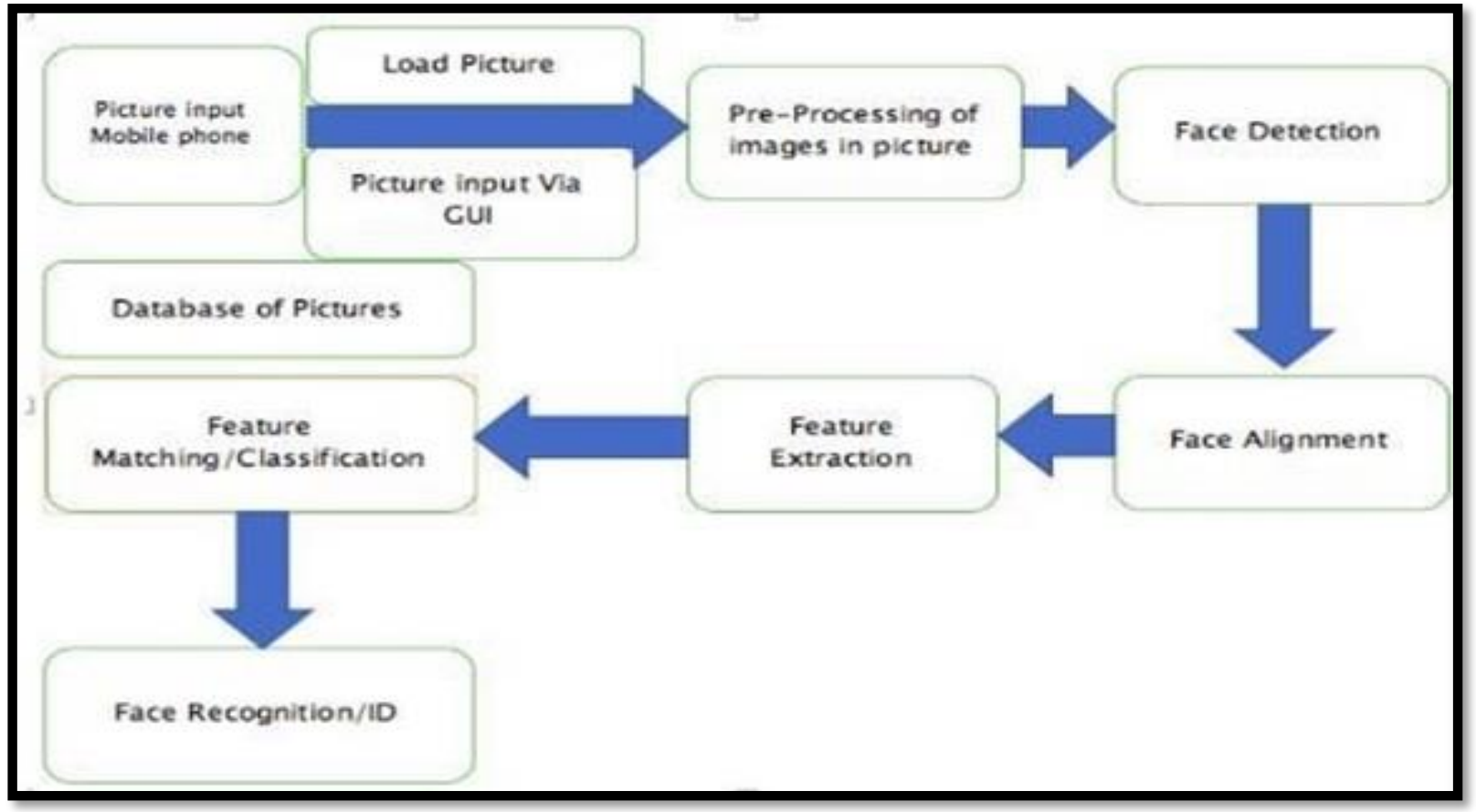

Fig.5.3 Flow Diagram of Proposed Working Model

DOI Number: https://doi.org/10.30780/specialissue-ICACCG2020/0033

Paper Id: IJTRS-ICACCG2020-033

pg. 73

@ 2017, IJTRS All Right Reserved, www.ijtrs.com 
ICACCG2020 30-31 July, 2020, Ansal University, Gurgaon, India

International Journal of Technical Research \& Science (Special Issue)

ISSN No.:2454-2024 (online)

6. COST ESTIMATION

\begin{tabular}{|c|c|c|}
\hline S.No. & Component & Price (₹) \\
\hline 1. & Raspberry Pi & ₹ 2,500 \\
\hline 2. & Camera & ₹ 500 \\
\hline 3. & LED Display Board & ₹ 500 \\
\hline 4. & Jumping Wires & ₹ 100 \\
\hline & Total & ₹ $\mathbf{3 6 0 0}$ \\
\hline
\end{tabular}

Table 6.1 Cost Estimation of Proposed Working Model

\section{CONCLUSION}

Thus, the aim of this project is to recognize the face of students and match it to the database to ensure their presence or absence, mark attendance to the particular student to maintain the record. And the smart board displays the total strength of the student in the class and how many students are present in the current time. The Smart Display board with CCTV Attendance System helps in increasing the accuracy and speed ultimately achieve the high-precision realtime attendance to meet the need for automatic classroom attendance.

Advantages-

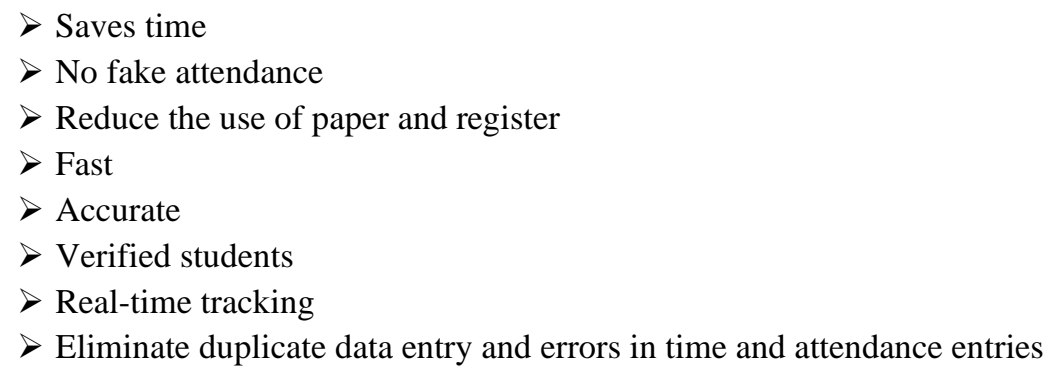

Capturing the images from the camera or CCTV camera and applying techniques face detection and recognition can decrease the manual work from human and increase the security safety, taking the decision from this recognition result. Based on this face detection and recognition can be used in implement so many applications like automatic attendance system based on face recognition, worker attendances, security, safety, police application like finding a thief in the image that helps to catch the thief. In this system, we have implemented an attendance system for a lecture, section or laboratory by which lecturer or teaching assistant a record student's attendance. It saves time and effort, especially if it is a lecture with a huge number of students.

\section{REFERENCES}

[1] Raspberry Specification - https-//www.raspberrypi.org/products/raspberry-pi-3-model-b/

[2] Kosov S., Scherbaum K., Faber K., Thormahlen T., and Seidel H.-P., "Rapid stereo-vision enhanced face detection", Image Processing (ICIP), 2009 16th IEEE International Conference on, IEEE, 1221-1224 (2009)

[3] Zhao W., Krishnaswamy A., Chellappa R., Swets D. L., and Weng J., "Discriminant analysis of principal components for face recognition", Face Recognition, Springer 73-85. (1998)

[4] Kanade T., "Picture processing system by computer complex and recognition of human faces", (1974)

[5] Levada A., Correa D., Salvador D., Saito J., and Mascarenhas N., "Novel approaches for face recognitiontemplate matching using dynamic time warping and LSTM NeuralNetwork Supervised Classification", Systems, Signals, and Image Processing, 2008. IWSSIP 2008. 15th International Conference on, IEEE, 241-244 (2008)

[6] Face Recognition Based Attendance System by Nandhini R, Duraimurugan N, S.P.Chokkalingam at International Journal of Engineering and Advanced Technology (IJEAT) ISSN- 2249 - 8958, Volume-8, Issue3S, February 2019.

[7] T.Kanade, "Picture processing by computer complex and recognition of human faces," technical report, Dept. Information Science, Kyoto Univ., 1973.

[8] Attendance System based on Face Recognition by Venkata Kalyan Polamarasetty at International Research Journal of Engineering and Technology (IRJET) Volume- 05 Issue- 04 | Apr-2018.

[9] Face Detection and Recognition Student Attendance System-

[10] https//www.researchgate.net/publication/326986115_Face_Detection_and_Recognition_Student_Attendance_ System 